\title{
A Study on the Rural Migrant Worker's Citizenization in the New Urbanization from the Perspective of New Normal Development ${ }^{1}$ Yi-ping SUN ${ }^{1, *}$ and Xiao-jun SUN ${ }^{2}$ \\ ${ }^{1}$ Department of Business Administration, Hubei University of Economics, Wuhan, 430205, China \\ ${ }^{2}$ School of Foreign Languages, Hubei University of Economics, Wuhan, 430205, China
}

Keywords: New normal, New urbanization, Rural migrant worker's citizenization.

\begin{abstract}
Urbanization, as the main driving force of national modernization, serves as the only way for economic and social development. In accordance with the law of economic and social development, China's economy has entered the "new normal" stage of medium and high-speed growth. According to the law of urbanization development in the world, China has entered a new stage where expanding domestic demand and reform dividend through new urbanization, has been the main concern, so does the rural migrant worker's citizenization of "new normal" development. However, due to lagging level of urban economic development, limited carrying capacity, related institutional barriers and system defects, lack of relevant safeguard measures, as well as self-quality and the general public prejudice and other reasons currently lead to the stagnation of rural migrant worker's citizenization. Based on the analysis of the causes of citizenization of rural migrant workers, this paper analyzes the basic principles of rural migrant workers' citizenization in the new urbanization, and puts forward some countermeasures and suggestions gaining the support from economic security, policy system, convenience and culture for the rural migrant workers' citizenization in new urbanization in the new towns under the new normal conditions.
\end{abstract}

\section{Introduction}

Urbanization, as the main driving force of national modernization, serves as the only way for economic and social development. According to the law of urbanization development in the world, China has entered a new stage where expanding domestic demand and reform dividend, through new urbanization, has been the main concern. The central conference on urbanization work in 2013 pointed out that the guideline of urbanization, under the new normal conditions of economic development, should be shifted from "city-based" to "people-oriented". The National New Urbanization Program (2014-2020) in 2014 clearly points out that the new urbanization centers on people's urbanization and upgrading quality. In May 2015, the National Development and Reform Commission held a TV conference stressed that China's economic downward pressure gradually increased, needed steady growth in expanding domestic demand to tap the potential, strengthened innovation to increase vitality, and deepened the reform on dividends. The new urbanization both can not only expand consumer demand, but also stimulate investment demand, displaying itself as integrated platform to promote innovation and entrepreneurship as well as deepen the reform.

${ }^{1}$ This research was financially supported by Hubei Corporate Culture Research Center 2015 Program (Program No. 2015B01). 
Therefore, we should give full play to the new urbanization to balance growth, promote reform, and adjust the structure, which has positive effect on livelihood. Urbanization involves five elements, i.e. people, industry, money, land and housing, in which people is the key part in line with the "people-oriented" thought from the scientific concept of development. For example, the 2014 Government Work Report emphasize show to solve the problem of "three hundred million people" in the process of new urbanization, that is, to work out the transfer of about 100 million agricultural population to settle in cities and towns; to renovate of about 100 million households"' urban shanty towns and villages; to guide about 100 million people in the central and western regions towards the urbanization. In June 2015, the central comprehensive reform of the Leading Group adopted the "further promoting the reform of the household registration system views." Based on the current economic situation at home and abroad as well as the economic characteristics in "new normal" development, how to adapt to the "new normal" and lead the "new normal" has recently become scholars ' top concern.

Throughout the course of urbanization in China, urbanization has moved tens of millions of rural people to the city, bringing enormous changes. According to the National Bureau of Statistics data, each year a large number of rural surplus labor flock into urban employment or non-agricultural employment population. By the end of 2014 the total number of rural migrant workers in China has reached 270 million, while China's resident population urbanization rate has exceeded by $52 \%$. The urbanization rate of population is only $35 \%$, which shows that more than 200 million rural migrant workers have changed from rural to urban areas and from agriculture to non-agricultural, but they have not realized the change from farmers to citizens. These rural migrant workers in the field of employment, involving many industries, are an important force in China's modernization drive, and have an irreplaceable and important contribution. But what people cannot ignore is that the urbanization is followed by a larger gap between urban and rural areas. The ecological environment has been damaged; migrant workers cannot enjoy the "public" treatment, free in the urban fringe. Although they have been engaged in non-agricultural industry employment, long-term residence in the city, but the disadvantaged position is very obvious. The typical feature is that migrant workers cannot obtain urban Hukou, and cannot enjoy urban public services and social welfare. Thus, in the "new normal" context, how to achieve the citizenization of migrant workers in urbanization becomes a top priority. According to the Report on the Process of Citizenization of Rural Migrant Farmers in China (2012), despite the improvement of cultural quality, both from public service and economic life, the rural migrant workers' treatment, in all aspects of the urbanization, has not been significantly improved, so the process of urbanization of migrant workers stalls. The urbanization of rural migrant workers lags behind, which is not conducive to the healthy development of China's urban economy, and may have an important impact on social harmony. Therefore, it is of great theoretical and practical significance to promote the citizenization of rural migrant workers and the healthy development of China's urban economy, break the urban-rural dual system, and coordinate urban and rural development. 


\section{The Connotation of the Relationship between the New Urbanization and the Citizenization of Rural Migrant Workers upon the "New Normal"}

\section{New Connotation of Urbanization under the "New Normal"}

"New normal" refers that China's economic growth rate slows down after years of high-speed development, focusing on improving the quality of economic development stage characteristics (Han Shuo and Dai Yue, 2015). In the "new normal" context, China's economic development pays more attention to quality improvement, then the new urbanization is bound to require the development of medium-speed under the premise of focusing on urbanization level of quality. The new urbanization "new" in the adherence to the scientific concept of development of the "people-oriented", that is, from the initial "city-based" to "people-oriented" change, and correctly guide the rural population mobility, promote agricultural population, increase public service coverage. We should pay attention to improving the quality of the population and promoting social fairness and justice. We should also handle the relationship between social development and ecological environment, stick to the road of sustainable development and adhere to the road of urbanization with Chinese characteristics (Li Chenghua 2015). The new urbanization itself has the characteristics of scientific development concept, coordinated development process, intensive development mode, and human development goal (Wang Suzhai, 2013). On the one hand, the macroeconomic policy in guiding economic transition also requires the development of urban areas in various fields to make the appropriate reform, namely, economic growth model (1) economic growth model and the transformation of urbanization model to a certain degree of coherence, Changes will affect the development trend of urbanization and progress. On the other hand, the policies in the process of urbanization can support the transformation of sustainable and high efficiency oriented economic growth model (Zhang Wanhang, 2015). The new urbanization under the new normal needs to give full play to the decisive role of the market, adhere to the people-oriented, to the community to provide high-quality public services, reasonable urban and rural development.

\section{New Connotation of Citizenization of Rural Migrant Workers under the "New Normal"}

Citizenship of rural migrant workers refers to the process of how rural migrant workers get legal identity and social rights, as promoted by the laws and policies of the state (Cheng Shu, 2013). China's urban-rural dual system and the existence of household registration system make the transfer of rural migrant workers to the city extremely difficult, that is, the process of Chinese farmers, unlike the foreign countries, is not only a single conversion from farmers to the citizen, and need a indispensable step i.e.rural migrant workers. Therefore, the process of citizenization of rural migrant in China is of Chinese characteristics.

\section{Relationship between the New Urbanization and the Citizenization of Rural Migrant Workers upon the "New Normal"}

The essence of urbanization is to realize the transfer of population from rural to urban areas. The ultimate aim of new urbanization is to realize the all-round development of human beings, and insist on people-oriented and realize urbanization. "New normal" under the new urbanization is to uphold the people-oriented, to ensure the realization of high-quality urbanization. "People-oriented" in the process of urbanization mainly 
refers to the people, mainly rural migrant workers, and then the main problem of new urbanization is to solve the problem of rural migrant workers. Citizenization of rural migrant workers is the main task of new urbanization, and only an effective solution to rural migrant workers in the city can truly realize the new urbanization.

\section{The Undergoing Plight of the Citizenization of Rural Migrant Workers in the New Urbanization}

The main obstacle can be attributed to the following: weak economic foundation, institutional barriers and system defects, lack of relevant security measures, low quality of rural migrant workers and cognitive bias.

\section{The Limited Carrying Capacity Due to the Lagging Development of Urban Economy}

From the urban development S-curve theory, we can see that the process of urbanization in China is still in the middle stage, which is characterized by the rapid expansion of urban scale, the increasing effect of urban agglomeration, and more and more rural surplus labor force into town. At the same time, it can not be ignored that at this stage, the infrastructure and public resources of the city have not increased, such as land, water resources and roads, which are difficult to meet the rapid growth of the needs of foreign population, especially in some mega-cities. In other words, citizenization of rural migrant workers lacks a strong economic base.

\section{Institutional Barriers and Deficiencies}

Urban-rural dual system leads to the "double marginalization" position of rural migrant workers. Household registration system is an important feature of the dual system of urban and rural areas, for rural surplus labor, leaving the land to migrant workers is only the realization of the transfer of occupation and geographical identity without any changes in the characteristics of urban identity. Being unable to get Hukou has been an important obstacle for them. The lack of thoroughness of the citizens has brought the separation of rural migrant workers' status, that is, they have been an important part of the construction workers in their occupation, but the identity is still the peasant. Under the current situation, the urban and rural employment mechanism is unequal, the labor market is far from integration, the rural surplus labor is difficult to obtain comprehensive employment information and equal employment environment, it is difficult to obtain formal employment jobs in the city, and cannot integrate into the mainstream society, forming a vicious circle. Their job mobility is simply the transformation of labor positions, the lack of continuous accumulation, there is no stable income, and thus do not have the ability to translate into urban population. In addition, from an external perspective, the dual system of social security system cannot take equal care of migrant workers. The city's high housing prices are beyond the affordability of rural migrant workers; their children cannot participate in the college entrance examination; many factors make them marginalized. In this case, the rural land has become the last protection of rural migrant workers, and their last retreat of the home, the subjective cannot be separated from the land of contact, but also makes it difficult to complete the citizens.

\section{The Lack of Relevant Safeguards}

Citizenization of rural migrant workers needs strong government policy guidelines, and even that the government should play a leading role. However the actual situation is that the relevant policy efforts are not in place. From the central government level, 
currently the special policy system has not yet formed to solve the problem of rural migrant workers. At present, the relative policies are concentrated in the "State Council on the issue of resolving the issue of rural migrant workers a number of opinions", the rest of the policy is more dispersed, lacking of strong legal protection and long-term planning. In addition, the incompleteness and inequality of the urban public service system also make the rural migrant workers unable to enjoy the same public services as the citizens and cannot be integrated into the urban community and the ecological environment effectively. From the local government level, the formulation of local policies is often discriminatory, focusing only on the interests of the local people in the management of rural migrant workers and the public different treatment. Local cities face the problems of shortage of urban resources, limited capacity and insufficient public services. They are often unable to bear the high conversion cost of rural migrant workers' citizenship. Therefore, there is a phenomenon of economic absorption and policy exclusion. This man-made management tilts, so that rural migrant workers encounter political, economic, cultural, social security, medical, education and other aspects of inequality, seriously hindering the process of its citizenization.

\section{Self-quality of Rural Migrant Workers needs Further Improvement}

At present, many external factors have a certain negative impact on the process of rural migrant workers' citizenization, but from the perspective of rural migrant workers, that is, internal factors, the quality of rural migrant workers needs to be further improved. Rural migrant workers cannot be simply defined as cheap labor, but should create conditions to improve their human capitals and social capitals, because these factors affect their access to citizenship. From the actual situation, most of the rural migrant workers engaged in jobs are mainly in the labor-intensive industries. Their vocational skill is low, which is mainly due to their low level of education. Although the cultural level of Cenozoic migrant workers has been greatly improved with the popularization of nine-year compulsory education, they still lack the basic skills and experience needed to obtain stable jobs in cities. At present, the most demanded labor market in the city is precisely the specialized labor education with certain professional skills. It is obvious that the rural migrant workers' skill level has affected the process of citizenization.

\section{Awareness of Prejudice Hinders Citizenization of Rural Migrant Workers}

Urban and rural development system makes the difference between urban residents and rural migrant workers in the cognitive gap, and even psychological distance. Social life is also common in urban residents of rural migrant workers groups who do not understand or respect individual places also appear to exclude foreign population phenomenon. On the one hand, the process of urbanization requires rural migrant workers, but on the other hand, urban residents only regard them as cheap labor, and do not allow them to enjoy equal treatment with urban residents, and even ignore their legitimate rights and interests. This has led rural migrant workers groups easily feel heart imbalance, being more difficult to integrate into the town. In this regard, only the government, the media, the general public work together to abandon prejudice and from an equal perspective on rural migrant workers, as well as accept them from the heart in order to make them easily into the social environment. 


\section{The Basic Principles of Citizenization of Rural Migrant Workers in the New Urbanization}

\section{Take into Account Differences and Diversification}

The process of citizenization cannot be achieved overnight, so we must consider the group differences, the level of the degree of improvement of the people. Employment stability is an important factor in the process of citizenization, so the degree of citizenization should be treated differently according to the employment situation of rural migrant workers. Specifically, medical education, family planning and other basic rights and interest's protection should be fully liberalized, and not affected by the stability of the impact of employment. For the minimum life, housing security, it should be mainly targeted at a group of rural migrant workers with more employment. According to the actual capacity of each local city, it may consider this group of migrant workers into the minimum living and housing security, and gradually expand the proportion of the gradual inclusion. Moreover, the basic social insurance, combined with their own peasant workers, should do targeted adjustments, as much as possible to cover a wider range of rural migrant workers groups.

Rural migrant workers should take a variety of urbanization initiatives. For small and medium-sized cities and towns, the city ,itself is in the urban and rural areas, should be the main acceptance of rural migrant workers, can consider the "open household" restrictions, encourage and support the local surplus rural labor in situ transformation. For large and medium cities, they should focus on opening the household registration restrictions to local migrant workers and creating conditions for its integration. At present, some large and medium-sized cities have begun to explore the establishment of a unified urban and rural old-age medical insurance system, which is expected to narrow the gap between urban and rural development, rural migrant workers groups to liberalize household registration restrictions are more likely. For some large cities where public resources are close to saturation, it is not suitable for large-scale release of the current restrictions on rural migrant workers, you can give some of the treatment of rural migrant workers, the formation of a transition zone. Combining with the present situation of the economic cascade development in eastern, central and western China, it is necessary to distinguish rural migrant workers from rural migrant workers in different regions and absorb the local rural migrant workers' groups.

\section{Adhere to the Overall Promotion and Moderate Scale}

"Three Rural Issues" is a long-term and arduous problem in China. The solution of the "three rural issues" is of great significance to the advancement of China's modernization drive. But the reality is that China's agricultural population is huge, and per capita land area is insufficient, so only relying on agriculture itself to solve the "three rural issues" is clearly difficult to complete. Therefore, unified development between urban and rural places is an effective solution. In this process, the farmers turn into urban citizens, being the core of urban and rural development as well as solution towards the "three rural issues" the fundamental point. Promoting urbanization is also an important measure to expand domestic demand and promote consumption, a large number of rural surplus labors transferred to the city, which naturally have impact on infrastructure, public services, housing construction needs. At present, China's rural population is not strong enough to consume, the quality of consumption is not high, the concept of consumption is backward, and there is a big 
gap with the urban residents. Once the rural population is converted into urban population, under the premise of solving the stable employment, this group naturally becomes the new force of consumption, and lifestyle and consumption patterns will have a significant upgrade. At the same time with the transfer of rural surplus labor force in the conditional agglomeration of land area, per capita operating land size will be greatly improved, which is to promote rural land intensive production and promotion of mechanized operations of great benefit will accelerate the "three rural issues" of the solution. Therefore, from this point of view, the citizenization of rural migrant workers, as the only way for promoting China's modernization play an important role in the expansion of domestic demand, adjustment of consumption structure, promotion of national economic development. However, it should be noted that the process of citizenization of rural migrant workers must be commensurate with the pace of economic development within the scope of the city can accommodate if China's citizenization needs of rural migrant workers base and fast growth cause ecological environment pressure, social problems will be exposed such as environmental pollution, traffic congestion, the gap between rich and poor, poor living conditions and other.

\section{Countermeasures and Suggestions for the Rural Migrant Workers' Citizenization in New Urbanization}

In the process of new urbanization, the rural migrant workers' citizenship should be integrated, and the new urban development system should be constructed to adjust the industrial structure, promote the upgrading of industrial structure and strengthen the construction of urban basic service facilities. Through a number of measures, coordinated development, are needed in order to address the process of urbanization of rural migrant workers to provide solutions for the stagnation.

\section{Adhere to the "Four Simultaneous" as Economic Support}

The new type of urbanization is to absorb the process of floating population from rural to urban places while the ability of cities and towns to absorb rural labor force is the result of mutual promotion between urbanization and industrial structure. That is, the industrial structure of cities and towns to a certain extent determines the level of urban surplus labor to absorb rural areas (Pu Yanping, Wu Yongqiu, 2005; Deng Zhituan, but Tao-Bo, Zeng Xiangquan, Chen Liwen, Yang Yumei, 2013) The 18th National Congress of the Communist Party of China put forward the "four modernizations", that is, insisting on Chinese characteristics, taking the road of industrialization, urbanization, informatization and agricultural modernization. Under the "new normal", China's economic development have moved into transition and the new urbanization also need to adjust the industrial structure and industrial upgrading, through the modern manufacturing and service industries for the new urbanization to provide a powerful driving force. The new urbanization development opportunity under the "four modernizations" concept and "new normal state", and reasonable coordination of the relationship between industrialization, urbanization, informationization and agricultural modernization, is to provide economic guarantee for rural migrant workers' citizenization. First of all, agricultural modernization is the foundation. Urbanization promotes the development of agricultural modernization, and agricultural modernization drives urbanization level (Xu Xiaojun, 2015). Agricultural modernization can, to the greatest extent, liberates the rural labor force and provides more surplus labor force for urbanization, which is an important source 
of labor force for urban economic development. Agricultural modernization makes more farmers have the opportunity to enter the city, start a new life for rural migrant workers to provide an opportunity for the citizens. Second, industrialization is the driving force. Industrialization can effectively solve the rural surplus labor force brought by agricultural modernization, and provide financial and technical support for the sustainable and healthy development of the economy and urbanization. Third, the information is guaranteed. Informatization can promote the optimization of urban industrial structure audit, and change the mode of economic development, the use of scientific and technological innovation, urbanization and the provision of information technology. Informatization can provide information technology support to the citizens in the process of rural migrant workers' citizenization, and ensure the sustainable transitivity of social security information. Therefore, only by insisting on agricultural modernization, industrialization and the simultaneous development of information technology and urbanization can we ensure that the level of economic development in the process of urbanization has improved. Economic base determines the superstructure, adhere to the "four simultaneous", and vigorously develop the urban economy, to be able to rural migrant workers for the implementation of public policy to provide economic security.

\section{Build a New Institution of Urban Development as System Support}

The process of urbanization of rural migrant workers is very difficult due to the problems of subsistence, residence, population transfer, rights protection, group integration and role transformation. In particular, China is still under the dual development of urban and rural areas, many institutional and institutional obstacles to be eliminated. China is in a critical period of deepening reform, and the importance of migrant workers is self-evident. We must build a new system of urban development, combined with a number of measures introduced policies and systems, actively and steadily forward. First of all, in the new urbanization development system, deepen the reform of the household registration system, followed by the corresponding supporting system reform. Practice has proved that the key to reforming the household registration system is to change the inequality brought by the other binary systems embedded in the household registration system (Huang, 2009). This shows that the establishment of a sound system to deepen the reform of the household registration system is very necessary. Therefore, the "new normal" under the new citizenization of rural migrant workers to solve the problem of urbanization must also rely on the corresponding social security system, employment system.

Deepening the Reform of Household Registration System to Achieve Urban and Rural Unity. Many developing countries control their domestic migration through policy implementation (World Bank, 1984). China, of course, is no exception. Population policies are used to control the influx of people from outside the region, and the household registration system is used to implement regional differential policies. Since the reform and opening up, China began the binary household registration system for progressive reform, but because the focus of household registration reform is to attract capital, technology and talent, rather than the real population free migration, household registration system reform is limited to the household registration. The relevant welfare system does not keep up with the same time, different local household registration reform styles; lack of unified planning and so on, the above reasons led to the reform of China's household registration system is slow (Huang Kun 2009). China's current reform of the household registration system is not complete, on the one hand, reduce the willingness of migrant workers, on the 
other hand to improve the threshold and cost of migrant workers, so to fundamentally solve the problem of migrant workers, the central government needs to develop a unified household registration reform program to break the urban and rural dual economic structure, whether in education, housing or social security to achieve equal treatment of the standard to achieve urban and rural unity, and fundamentally remove barriers for migrant workers.

Improve the Social Security System and Expand the Coverage of the Social Security System. For the perfection of the social security system of rural migrant workers, we must consider the actual needs of rural migrant workers, and also the city's own conditions. It should be noted that the social security system for rural migrant workers is an all-round system, with medical, pension and work injury insurance being the most necessary (Zhang Tai-yu, 2014). Only by satisfying these three basic needs can an effective social security system be built, so as to gradually implement the mobility of migrant workers. The social security system of rural migrant workers has been explored in some areas. However, due to its low level and unstable operation, the social security system has been affected by the social security system. In addition, it has not taken into account the frequent mobility of rural migrant workers, such as social security funds and premiums, regional transfer and so on. From this point of view, it's a must for authoritative institutions from the national unity level of top-level design, central unified scheduling, and unified supervision, to formulate standard operating mode and norms for rural migrant workers on social security fund payment, withdrawal, cross-regional transfer. At the same time, the central government should also increase financial investment, set up an effective social security service agencies, urge the corresponding laws and regulations promulgated, the specific implementation of the supervision, publicity and popularization. The local government should make full use of the characteristics of temporary and mobility of migrant workers in informal employment according to their own conditions and local conditions, strengthen the construction of social security information, and facilitate the transfer of social insurance relationship for rural migrant workers. The government has allocated more social security resources to the urban residents in terms of the social security system, while rural migrant workers can only rely on household income to solve the problems of old age and medical care (White, 1998). This shows that the rights of the citizens and treatment of urban citizens are much higher than that of migrant workers (Solinger, 1999). The government should further expand the coverage of social security, to ensure that as many rural migrant workers can enjoy the full social insurance, to achieve social equity and build a harmonious society.

Unify Employment System to Promote Equal Employment. Rural migrant workers, as the mobility factor in the labor market, their free movement and access to equal employment opportunities for the full play to its own value has a crucial role. However, it should be noted that there are still obstacles to the free flow of migrant workers in current labor market, such as the common labor market segmentation and employment discrimination. Some cities have obvious local protectionism, in order to maximize the dissolution of the city laid-off workers employment pressure, or even simply to account for the threshold to limit the employment of rural migrant workers in a number of positions, thus forming a protective labor market. But economically, such a discriminatory local protectionist labor market has no significance in helping to expand employment. Instead, it will increase labor costs, limit labor quality, and improve market creation by limiting free competition, leading to a decline in economic competitiveness. "New normal" under the new urbanization is to give full 
play to the decisive role of the market, play a basic role in market allocation of resources. Thus, the Government should clarify the understanding, get rid of the dual employment system, do not ignore the rural migrant workers groups, but they should be given the same treatment. The specific approach is to dilute the Hukou and regional restrictions to eliminate the constraints of the free movement of labor barriers, to strengthen fundamental role of market in allocation of labor resources in and set the main position of the labor market, enabling migrant workers to equal participation in employment competition, its citizens to consolidate the material basis.

\section{Construct Urban Infrastructure of "People-Oriented" Services as Convenient Support}

The goal of new urbanization is to realize urbanization step by step on the basis of "people-oriented". The "people-oriented" should not only provide convenience for citizenization of rural migrant workers from the aspect of system policy and economic development, but also the basic service facilities housing supply in major cities, the rich urban housing supply, an effective solution to the problem of rural migrant workers housing difficult. Wang Feipeng (2015) pointed out that the unavailability of stable housing for rural migrant workers could not be neglected. According to Zhang Zhanbin (2013), the unstable housing aroused direct impact on the integrity of migrant workers in the family, the quality of life of rural migrant workers, health care, their children's education, and even many important social issues. The migrant workers' families cannot migrate to the city as a whole, but the individual short-term mobility is the main reason. Therefore, effort to solve the housing problem of rural migrant workers is particularly critical. But it is clear that the current high prices will be overwhelming for the majority of rural migrant workers, so what the government needs to do is to broaden channels, rich forms, and consider China's national conditions with income characteristics of rural migrant workers and income and expenditure to release restrictions on housing, improve their living conditions, and promote their citizenship. As early as 2007, the original Ministry of Construction, Ministry of Finance, Labor and Social Security, Development and Reform Commission, Ministry of Land and Resources jointly issued "guidance on improving the living conditions of rural migrant workers." The employment unite of the rural migrant workers should be more responsible for improving the living conditions of rural migrant workers, and provide housing subsidies, cheap rent or even free rent to improve or solve the housing problems of rural migrant workers considering specific circumstances. Employers bear the main responsibility for the provision of standardized housing for rural migrant workers, being the most direct and practical method to solve the practical difficulties, which in turn can be used as effective way to attract employment of rural migrant workers, strengthen the management of migrant workers, and ease social pressures. However, the nature of corporate pursuit of profitability and the mobility of migrant workers make this approach less longstanding to fundamentally solve the housing problem of rural migrant workers, so in the long run, professional social organizations and national institutions are needed to provide cheaper housing so as to improve their housing conditions.

\section{Build a Harmonious Social and Cultural Bridge as Cultural Support}

The new urbanization of "people-oriented" is in line with the purpose of building a socialist harmonious society. From the point of view of rural migrant workers, solving the problem of urbanization should not rely solely on the strength of external society. Although the government and social organizations have the obligation to clear away 
the external obstacles for migrant workers, and provide a lax institutional mechanism, the improvement of the quality of migrant workers is the key. We should guide rural migrant workers to improve self-academic ability, enhance cultural accomplishment, and strengthen the ability of psychological adaptation. From the perspective of the government, the media and the general public, we should fully realize the great contribution made by rural migrant workers to social and economic construction. They should abandon their prejudices and accept them from an equal angle, so as to create farmer-friendly working environment. With cultural services for rural migrant workers, we need rich their knowledge through providing technology-related lectures, building libraries and cultural centers in rural migrant workers' region.

\section{Conclusion}

Based on the analysis of the causes of citizenization of rural migrant workers, this paper analyzes the basic principles of rural migrant workers' citizenization in the new urbanization, and puts forward some countermeasures and suggestions gaining the support from economic security, policy system, convenience and culture for the rural migrant workers' citizenization in new urbanization in the new towns under the new normal conditions. Such as adhere to the "four simultaneous" as economic support, build a new institution of urban development as system support, construct urban infrastructure of "people-oriented" services as convenient support, and build a harmonious social and cultural bridge as cultural support.

\section{References}

[1] Cheng Shu. Urbanization of peasant workers in the process of urbanization [D]. Northeast Agricultural University doctoral dissertation, 2013.

[2] Deng Zhituan, but Tao wave. On the Rural Surplus Labor Transfer and Regional Industrial Structure Evolution [J]. China Rural Economy, 2005 (8): 30-36.

[3] Han Shuo, Dai Yue. Reflections on the Fiscal and Tax Policies to Promote the New Urbanization under the New Economic Norms,2015(11): 8-10.

[4] Huang Kun. Deepening the reform of household registration system and the peasant workers' urbanization [J]. Urban Development Research, 2009 (2): 97-104.

[5] Li Chenghua. (Social Sciences Edition) Journal of Nanjing University of Science and Technology (Social Sciences Edition) Journal of Nanjing University of Science and Technology (Social Science Edition)

[6] Pu Yanping, Wu Yongqiu. Economic Growth, Industrial Structure and Labor Transfer [J]. Quantitative Technology and Economic Research, 2005 (9): 19-29.

[7] Wang Feipeng. The Main Obstacles and Reform Measures of Peasant Workers into the City [J]. Contemporary Economic Management, 2015 (7): 63-67.

[8] Wang Suzhai. The connotation, goal and path of new urbanization scientific development [J]. Theory Monthly, 2013 (4): 1-7.

[9] Xia Jijun. Population Mobility, Public Income and Expenditure - Analysis on the Motivation of Household Registration System Change [J]. Economic Research, 2004 (10): 56-65. 
[10] Xu Xiaojun. Journal of Jianghan University (Social Science Edition), 2015 (1): 13-19 (in Chinese with English abstract).

[11] Have Xiangquan, Chen Liwen, Yang Yumei. Urbanization, Industrial Structure and the Efficiency of Rural Labor Transfer [J].

[12]Zhang Taoyu. Strengthening the investment of human capital and improving the ability of the peasant workers to participate in the insurance policy $[\mathrm{J}]$. Contemporary Economy, 2014 (22): 22-23.

[13]Zhang Wanhang. (3): 39-41 [J] China Development Review, 2015 (3): 39-41.

[14]Zhang Zhanbin. Journal of China National School of Administration, 2013 (1): 48-54. 